\title{
A NEW EXISTENCE THEORY FOR POSITIVE PERIODIC SOLUTIONS TO A NEUTRAL DELAY $n$-SPECIES COMPETITIVE MODEL WITH THE EFFECT OF TOXIC SUBSTANCE AND IMPULSE
}

\author{
JIE PENG, ZHENGUO LUO, YANG DENG, NI SHUAI \\ and PING LIU
}

School of Mathematics and Statistics

Hengyang Normal University

Hengyang, Hunan 421002

P. R. China

e-mail: robert186@163.com

\begin{abstract}
In this paper, we acquire some sufficient and realistic conditions for the existence of positive periodic solution for a general neutral impulsive $n$-species competitive model with the effect of toxic substance and infinite delay, by applying some analysis techniques and a new existence theorem, which is different from Gaines and Mawhin's continuation theorem and abstract continuation theory for $k$-set contraction. As an application, we also examine some special cases which have been studied extensively in the literature. Some known results are improved and generalized.
\end{abstract}

2010 Mathematics Subject Classification: 35C25, 34D20, 37N25.

Keywords and phrases: periodic solution, neutral, infinite delay, $n$-species competitive model, impulse.

Received March 10, 2017

(ㄷ) 2017 Scientific Advances Publishers 


\section{Introduction}

In this paper, we investigate the existence of the positive periodic solution of the following $n$-species competition system with general periodic neutral delay and impulse:

$$
\left\{\begin{aligned}
N_{i}^{\prime}(t)= & N_{i}(t)\left[r_{i}(t)-\sum_{j=1}^{n} a_{i j}(t) N_{j}(t)-\sum_{j=1}^{n} b_{i j}(t) \int_{-\infty}^{t} k_{i j}(t-s) N_{j}(s) d s\right. \\
& -\sum_{j=1}^{n} c_{i j}(t) N_{j}\left(t-\tau_{i j}(t)\right)-\sum_{j=1}^{n} d_{i j}(t) N_{j}^{\prime}\left(t-\gamma_{i j}(t)\right) \\
& \left.-\sum_{j=1}^{n} e_{i j}(t) N_{i}(t) N_{j}(t)\right], i=1,2, \ldots, n, t \neq t_{k}, \\
\Delta N_{i}\left(t_{k}\right) & =\left(p_{i k}+q_{i k}\right) N_{i}\left(t_{k}\right), i=1,2, \ldots, n, \quad k=1,2, \ldots,
\end{aligned}\right.
$$

with initial conditions:

$$
\begin{aligned}
& N_{i}(\xi)=\varphi_{i}(\xi), \quad N_{i}^{\prime}(\xi)=\varphi_{i}^{\prime}(\xi), \quad \xi \in[-\tau, 0], \quad \varphi_{i}(0)>0, \\
& \varphi_{i} \in C\left([-\tau, 0), R^{+}\right) \bigcap C^{1}\left([-\tau, 0), R^{+}\right), i=1,2,3, \ldots, n,
\end{aligned}
$$

where $a_{i j}, b_{i j}, c_{i j}, e_{i j} \in C(R,[0,+\infty)), d_{i j} \in C^{1}(R,[0,+\infty)), \tau_{i j} \in C^{1}(R, R)$, $\sigma_{i j} \in C^{2}(R, R)$ are continuous $\omega$-periodic functions. $r_{i} \in C(R, R)$ are continuous $\omega$-periodic functions with $\int_{0}^{\omega} r_{i}(t) d t>0$. The growth functions $r_{i}$ are not necessarily positive, since the environment fluctuates randomly, in some conditions $r_{i}$ may be negative. $\tau=\max _{1 \geq i \geq n, 1 \geq j \geq n}$ $\left\{\max _{t \in[0, \omega]}\left\{\tau_{i j}(t), \gamma_{i j}(t)\right\}\right\} \cdot \int_{0}^{\infty} k_{i j}(s) d s=1, \int_{0}^{+\infty} s k_{i j}(s) d s<+\infty, i, j=1,2, \ldots, n$. And, $p_{i k}$ and $q_{i k}$ represent the birth rate and the harvesting (or stocking) rate of $N_{i}$ at time $t_{k}$, respectively. When $q_{i k}>0$, it stands for harvesting, while $q_{i k}<0$ means stocking. For the ecological justification of (1.1) and the similar types refer to [1-6]. 
In 1993, Kuang in [1] proposed an open problem (Open problem 9.2) to obtain sufficient conditions for the existence of a positive periodic solution of the following equation:

$$
\frac{d N}{d t}=N(t)\left[a(t)-\beta(t) N(t)-b(t) N(t-\tau(t))-c(t) N^{\prime}(t-\tau(t))\right] .
$$

In [2], Fang and Li studied Equation (1.3) and gave an answer to the open problem 9.2 of [1]. But paper [2] required that $b(t) \geq 0, c(t) \geq 0$ and $c_{0}^{\prime}(t)>b(t), \beta(t) \geq 0$ or $c_{0}^{\prime}(t) \leq b(t), \beta(t) \leq 0$ for $t \in[0, \omega]$, where $c_{0}(t)=\frac{c(t)}{1-\tau^{\prime}(t)}$.

In [3], Lu and Ge investigated a neutral delay population model with multiple delays:

$$
\frac{d N}{d t}=N(t)\left[a(t)-\beta(t) N(t)-\sum_{j=1}^{n} b_{j}(t) N\left(t-\sigma_{j}(t)\right)-\sum_{i=1}^{m} c_{i}(t) N^{\prime}\left(t-\tau_{i}(t)\right)\right]
$$

they applied the theory of abstract continuous theorem of $k$-set contractive operator and some analysis techniques to obtain some sufficient conditions for the existence of positive periodic solutions of the model (1.4).

For multiple species system, in [4], Yang and Cao investigated the following a general neutral Lotka-Volterra system with unbounded delays:

$$
\begin{aligned}
\frac{d N_{i}}{d t}= & N_{i}(t)\left[a_{i}(t)-\sum_{j=1}^{n} \beta_{i j}(t) N_{j}(t)-\sum_{j=1}^{n} b_{i j}(t) N_{j}\left(t-\tau_{i j}(t)\right)\right. \\
& \left.-\sum_{j=1}^{n} c_{i j}(t) N_{j}^{\prime}\left(t-\gamma_{i j}(t)\right)\right], i=1,2, \ldots, n .
\end{aligned}
$$

Author applied the theory of coincidence degree to obtain verifiable sufficient conditions of the existence of positive periodic solutions of system (1.5). 
Recently, Liu and Chen [5] further discussed the above system (1.5). They introduced a new existence theorem to obtain a set of sufficient conditions for the existence of positive periodic solutions for system (1.5), improved and generalized some known results.

On the other hand, there are some other perturbations in the real world such as fires and floods, that are not suitable to be considered continually. These perturbations bring sudden changes to the system. Systems with such sudden perturbations involving impulsive differential equations have attracted the interest of many researchers in the past twenty years [6-10], since they provide a natural description of several real processes subject to certain perturbations whose duration is negligible in comparison with the duration of the process. Such processes are often investigated in various fields of science and technology such as physics, population dynamics, ecology, biological systems, optimal control, etc. For details, see [11-15]. Recently, some qualitative properties such as oscillation, asymptotic behaviour, and stability are has been studied extensively by many authors [16-22].

In [19], Xia studied the following neutral impulsive delayed LotkaVolterra competition system with the effect of toxic substance:

$$
\left\{\begin{aligned}
& N_{i}^{\prime}(t)=N_{i}(t)\left[r_{i}(t)-\sum_{j=1}^{n} a_{i j}(t) N_{j}(t)-\sum_{j=1}^{n} b_{i j}(t) N_{j}\left(t-\tau_{i j}(t)\right)-\sum_{j=1}^{n} c_{i j}(t) N_{j}^{\prime}\left(t-\gamma_{i j}(t)\right)\right. \\
&\left.-\sum_{j=1}^{n} e_{i j}(t) N_{i}(t) N_{j}(t)\right], i=1,2, \ldots, n, t \neq t_{k}, \\
& \Delta N_{i}\left(t_{k}\right)=\left(b_{i k}+h_{i k}\right) N_{i}\left(t_{k}\right), i=1,2, \ldots, n, \quad k=1,2, \ldots,
\end{aligned}\right.
$$

by using some techniques of Mawhin coincidence degree theory, he obtained sufficient conditions for the existence of periodic positive solutions of the system (1.6). 
In [20], Wang and Dai investigated the following periodic neutral population model with delays and impulse:

$$
\left\{\begin{aligned}
\frac{d N}{d t}= & N(t)\left[a(t)-e(t) N(t)-\sum_{j=1}^{n} b_{j}(t) N\left(t-\sigma_{j}(t)\right)\right. \\
& \left.-\sum_{i=1}^{m} c_{i}(t) N^{\prime}\left(t-\tau_{i}(t)\right)\right], t \neq t_{k}, \\
N\left(t^{+}\right)= & \left(1+\theta_{k}\right) N\left(t_{k}\right), k=1,2, \ldots,
\end{aligned}\right.
$$

they obtained some sufficient conditions for the existence of positive periodic solutions of the model (1.7) by using the theory of abstract continuous theorem of $k$-set contractive operator and some analysis techniques.

However, to this day, no scholars had done works on the existence of positive periodic solution of (1.1). One could easily see that system (1.3)-(1.7) are all special cases of system (1.1). Therefore, we propose and study the system (1.1) in this paper.

For the sake of generality and convenience, we make the following notation and assumptions: Let $\omega>0$ be a constant and

$C_{\omega}=\{x \mid x \in C(R, R), x(t+\omega)=x(t)\}$, with the norm defined by $|x|_{0}=\max _{t \in[0, \omega]}|x(t)|$;

$C_{\omega}^{1}=\left\{x \mid x \in C^{1}(R, R), x(t+\omega)=x(t)\right\}$, with the norm defined by $\|x\|=\max _{t \in[0, \omega]}\left\{|x|_{0},\left|x^{\prime}\right|_{0}\right\}$;

$P C=\left\{x \mid x: R \rightarrow R^{+}, \lim _{s \rightarrow t} x(s)=x(t)\right.$, if $t \neq t_{k}, \lim _{t \rightarrow t_{k}^{-}} x(t)=x\left(t_{k}\right)$, $\lim _{t \rightarrow t_{k}^{+}} x(t)$ exists; $\left.k \in Z^{+}\right\}$

$P C^{1}=\left\{x \mid x: R \rightarrow R^{+}, x \in P C\right\}$ 
$P C_{\omega}=\{x \mid x \in P C, x(t+\omega)=x(t)\}$, with the norm defined by $|x|_{0}=\max _{t \in[0, \omega]}|x(t)|$

$P C_{\omega}^{1}=\left\{x \mid x \in P C^{1}, x(t+\omega)=x(t)\right\}$, with the norm defined by $\|x\|=\max _{t \in[0, \omega]}\left\{|x|_{0},\left|x^{\prime}\right|_{0}\right\}$.

Then those spaces are all Banach spaces. We also denote

$$
\bar{h}=\frac{1}{\omega} \int_{0}^{\omega} h(t) d t, h^{M}=\max _{t \in[0, \omega]} h(t), h^{L}=\min _{t \in[0, \omega]} h(t), \text { for any } h \in P C_{\omega},
$$

$\Delta_{i j}=1+p_{i j}+q_{i j}$ and make the following assumptions:

$\left(\mathrm{H}_{1}\right)\left[t_{k}\right]_{k \in N}$ satisfies $0<t_{1}<t_{2}<\cdots<t_{k}<\cdots$ and $\lim _{k \rightarrow \infty} t_{k}=+\infty$;

$\left(\mathrm{H}_{2}\right)\left\{\Delta_{i k}\right\}$ is a real sequence such that $\Delta_{i k}>0, \prod_{0<t_{k}<t} \Delta_{i k}$, $l=1,2, \cdots, n$ are an $\omega$-periodic functions.

Definition 1.1. A function $N_{i}:[-\tau, 0] \rightarrow[0,+\infty), i=1,2, \cdots, n$ is said to be a positive solution of (1.1) and (1.2) on $[-\tau, \infty]$, if the following conditions are satisfied:

(a) $N_{i}(t)$ is absolutely continuous on each $\left(t_{k}, t_{k+1}\right)$;

(b) for each $k \in Z_{+}, N_{i}\left(t_{k}^{+}\right)$and $N_{i}\left(t_{k}^{-}\right)$exist and $N_{i}\left(t_{k}^{-}\right)=N_{i}\left(t_{k}\right)$;

(c) $N_{i}(t)$ satisfies the first equation of (1.1) and (1.2) for almost everywhere (for short a.e.) in $[0, \infty] \backslash\left\{t_{k}\right\}$ and satisfies $N_{i}\left(t_{k}^{+}\right)=\Delta_{i k} N_{i}\left(t_{k}\right)$ for $t=t_{k}, k \in Z_{+}=\{1,2, \cdots\}$.

Consider the non-impulsive delay differential equation:

$$
\begin{aligned}
y_{i}^{\prime}(t)= & y_{i}(t)\left[r_{i}(t)-\sum_{j=1}^{n} A_{i j}(t) y_{j}(t)-\sum_{j=1}^{n} B_{i j}(t) \int_{-\infty}^{t} k_{i j}(t-s) y_{j}(s) d s\right. \\
& \left.-\sum_{j=1}^{n} C_{i j}(t) y_{j}\left(t-\tau_{i j}(t)\right)-\sum_{j=1}^{n} D_{i j}(t) y_{j}^{\prime}\left(t-\gamma_{i j}(t)\right)-\sum_{j=1}^{n} E_{i j}(t) y_{i}(t) y_{j}(t)\right],
\end{aligned}
$$


with the initial conditions:

$$
\begin{aligned}
& y_{i}(t)=\phi_{i}(t), y_{i}^{\prime}(t)=\phi_{i}^{\prime}(t), t \in[-\tau, 0], \phi_{i}(0)>0, \\
& \phi_{i} \in C([-\tau, 0],[0, \infty)) \bigcap C^{1}([-\tau, 0],[0, \infty)),
\end{aligned}
$$

where

$$
\begin{gathered}
A_{i j}(t)=a_{i j}(t) \prod_{0<t_{k}<t} \Delta_{i k}, B_{i j}(t)=b_{i j}(t) \prod_{0<t_{k}<t} \Delta_{i k}, C_{i j}(t)=c_{i j}(t) \prod_{0<t_{k}<t-\tau_{i j}(t)} \Delta_{i k}, \\
D_{i j}(t)=d_{i j}(t) \prod_{0<t_{k}<t-\gamma_{i j}(t)} \Delta_{i k}, E_{i j}(t)=e_{i j}(t) \prod_{0<t_{k}<t} \Delta_{i k} \Delta_{j k}, \\
\tau=\max \left\{\tau_{i j}(t), \gamma_{i j}(t)\right\}, i, j=1,2, \cdots, n .
\end{gathered}
$$

The following lemmas will be used in the proofs of our results. The proof of the first lemma is similar to that of Theorem 1 in [6].

Lemma 1.1. Suppose that $\left(\mathrm{H}_{1}\right),\left(\mathrm{H}_{2}\right)$ hold, then

(1) if $y_{i}(t), i=1,2, \cdots, n$, is a solution of (1.8) and (1.9) on $[-\tau,+\infty)$, then $N_{i}(t)=\prod_{0<t_{k}<t} \Delta_{i k} y_{i}(t), i=1,2, \cdots, n$, is a solution of (1.1) and (1.2) on $[-\tau,+\infty)$.

(2) if $N_{i}(t), i=1,2, \cdots, n$, is a solution of (1.1) and (1.2) on $[-\tau,+\infty)$, then $y_{i}(t)=\prod_{0<t_{k}<t} \Delta_{i k}^{-1} N_{i}(t), i=1,2, \cdots, n$, is a solution of (1.8) and (1.9) on $[-\tau,+\infty)$. 
Proof. (1) It is easy to see that $N_{i}(t)=\prod_{0<t_{k}<t} \Delta_{i k} y_{i}(t)(i=1,2, \cdots, n)$ is absolutely continuous on every interval $\left(t_{k}, t_{k+1}\right] ; t \neq t_{k}, k=1,2, \cdots$,

$$
\begin{aligned}
& N_{i}^{\prime}(t)-N_{i}(t)\left[r_{i}(t)-\sum_{j=1}^{n} a_{i j}(t) N_{j}(t)-\sum_{j=1}^{n} b_{i j}(t) \int_{-\infty}^{t} k_{i j}(t-s) N_{j}(s) d s\right. \\
& \left.-\sum_{j=1}^{n} c_{i j}(t) N_{j}\left(t-\tau_{i j}(t)\right)-\sum_{j=1}^{n} d_{i j}(t) N_{j}^{\prime}\left(t-\gamma_{i j}(t)\right)-\sum_{j=1}^{n} e_{i j}(t) N_{i}(t) N_{j}(t)\right] \\
= & \prod_{0<t_{k}<t} \Delta_{i k} y_{i}^{\prime}(t)-\prod_{0<t_{k}<t} \Delta_{i k} y_{i}(t)\left[r_{i}(t)-\sum_{j=1}^{n} a_{i j}(t) \prod_{0<t_{k}<t} \Delta_{i k} y_{j}(t)\right. \\
& -\sum_{j=1}^{n} b_{i j}(t) \prod_{0<t_{k}<t} \Delta_{i k} \int_{-\infty}^{t} k_{i j}(t-s) y_{j}(s) d s-\sum_{j=1}^{n} c_{i j}(t) \prod_{0<t_{k}<t-\tau_{i j}(t)} \Delta_{i k} y_{j}\left(t-\tau_{i j}(t)\right) \\
& \left.-\sum_{j=1}^{n} d_{i j}(t) \prod_{0<t_{k}<t-\gamma_{i j}(t)} \Delta_{i k} y_{j}^{\prime}\left(t-\gamma_{i j}(t)\right)-\sum_{j=1}^{n} e_{i j}(t) \prod_{0<t_{k}<t} \Delta_{i k} \Delta_{j k} N_{i}(t) N_{j}(t)\right] \\
= & \prod_{0<t_{k}<t} \Delta_{i k}\left\{y_{i}^{\prime}(t)-y_{i}(t)\left[r_{i}(t)-\sum_{j=1}^{n} A_{i j}(t) y_{j}(t)-\sum_{j=1}^{n} B_{i j}(t) \int_{-\infty}^{t} k_{i j}(t-s) y_{j}(s) d s\right.\right. \\
& \left.\left.-\sum_{j=1}^{n} C_{i j}(t) y_{j}\left(t-\tau_{i j}(t)\right)-\sum_{j=1}^{n} D_{i j}(t) y_{j}^{\prime}\left(t-\gamma_{i j}(t)\right)-\sum_{j=1}^{n} E_{i j}(t) y_{i}(t) y_{j}(t)\right]\right\} \\
= & 0 .
\end{aligned}
$$

On the other hand, for any $t=t_{k}, k=1,2, \cdots$,

$$
N_{i}\left(t_{k}^{+}\right)=\lim _{t \rightarrow t_{k}^{+}} \prod_{0<t_{j}<t} \Delta_{i k} y_{i}(t)=\prod_{0<t_{j} \leq t_{k}} \Delta_{i k} y_{i}\left(t_{k}\right), \text { and } N_{i}\left(t_{k}\right)=\prod_{0<t_{j}<t_{k}} \Delta_{i k} y_{i}\left(t_{k}\right),
$$


thus

$$
\Delta N_{i}\left(t_{k}^{+}\right)=\Delta_{i k} y_{i}\left(t_{k}\right),
$$

which implies that $N_{i}(t)(i=1,2, \cdots, n)$ is a solution of (1.1) and (1.2). Therefore, if $y_{i}(t)(i=1,2, \cdots, n)$ is a solution of (1.8) and (1.9) on $[-\tau,+\infty)$, we can prove $N_{i}(t)(i=1,2, \cdots, n)$ are solutions of (1.1) and (1.2) on $[-\tau,+\infty)$.

(2) Since $N_{i}(t)=\prod_{0<t_{k}<t} \Delta_{i k} y_{i}(t)(i=1,2, \cdots, n)$ is absolutely continuous on every interval $\left(t_{k}, t_{k+1}\right] ; t \neq t_{k}, k=1,2, \ldots$, and in view of (1.11), it follows that for any $k=1,2, \ldots$,

$$
\begin{aligned}
y_{i}\left(t_{k}^{+}\right) & =\prod_{0<t_{j} \leq t_{k}} \Delta_{i k}^{-1} N_{i}\left(t_{k}^{+}\right)=\prod_{0<t_{j}<t_{k}} \Delta_{i k}^{-1} N_{i}\left(t_{k}\right)=y_{i}\left(t_{k}\right), \quad \text { and } \\
y_{i}\left(t_{k}^{-}\right) & =\prod_{0<t_{j}<t_{k}} \Delta_{i k}^{-1} N_{i}\left(t_{k}^{-}\right)=\prod_{0<t_{j} \leq t_{k}^{-}} \Delta_{i k}^{-1} N_{i}\left(t_{k}^{-}\right)=y_{i}\left(t_{k}\right), i=1,2, \ldots, n,
\end{aligned}
$$

which implies that $y_{i}(t)(i=1,2, \cdots, n)$ is continuous on $[-\tau,+\infty)$. It is easy to prove that $y_{i}(t)(i=1,2, \cdots, n)$ is absolutely continuous on $[-\tau,+\infty)$. Similar to the proof of (1), we can check that $y_{i}(t)=\prod_{0<t_{k}<t}$ $\Delta_{i k}^{-1} N_{i}(t)(i=1,2, \cdots, n)$ is a solution of (1.8) and (1.9) on $[-\tau,+\infty)$. The proof of Lemma 1.1 is completed.

From Lemma 1.1, if we want to discuss the existence and global asymptotic stability of positive periodic solutions of systems (1.1) and (1.2), we only discuss the existence of the existence and global asymptotic stability of positive periodic solutions of systems (1.8) and (1.9).

The organization of this paper is as follows. In the following section, we introduce some lemmas and an important existence theorem developed in $[23,24]$. In the third section, we derive some sufficient conditions which ensure the existences of positive periodic solution of system (1.1) by 
applying this theorem and some other techniques. Finally, we study some special cases of system (1.1) which have been studied extensively in the literature. These examples show that our sufficient conditions are new, and some known results can be improved and generalized.

\section{Preliminaries}

In this section, in order to obtain the existence of a periodic solution for system (1.8) and (1.9), we shall give some concepts and results from [24], state an existence theorem and some lemmas.

For a fixed $\tau>0$, let $C=: C\left([-\tau, 0] ; R^{n}\right)$. If $x \in C\left([-\tau, 0] ; R^{n}\right)$ for some $\delta>0$ and $\eta \in R$, then $x_{t} \in C$ for $t \in[\eta, \eta+\delta]$ is defined by $x_{t}(\theta)=x(t+\theta)$ for $\theta \in[-\tau, 0]$. The supremum norm in $C$ is denoted by $\|$.$\| , that is, \|\phi\|=\max _{t \in[-\tau, 0]}|\phi(\theta)|$ for $\phi \in C$, where $|$.$| denotes the norm$ in $R^{n}$, and $|u|=\sum_{j=1}^{n}\left|u_{i}\right|$ for $u=\left(u_{1}, \cdots, u_{n}\right) \in R^{n}$. Consider the following neutral functional differential equation:

$$
\frac{d}{d t}\left[x(t)-b\left(t, x_{t}\right)\right]=f\left(t, x_{t}\right)
$$

where $f: R \times C \rightarrow R^{n}$ is completely continuous and $b: R \times C \rightarrow R^{n}$ is continuous. Moreover, we assume:

(1) there exists $\omega>0$ such that for every $(t, \phi) \in R \times C$, we have $b(t+\omega, \phi)=b(t, \phi)$ and $f(t+\omega, \phi)=f(t, \phi) ;$

(2) there exists a constant $k<1$ such that $|b(t, \phi)-b(t, \varphi)| \leq k\|\phi-\varphi\|$, for $t \in R$ and $\phi, \varphi \in C$.

By using the continuation theorem for composite coincidence degree, Erbe et al. [23] proved the following existence theorem (see also Theorem 4.7.1 in [24]): 
Theorem A ([24]). Assume that there exists a constant $M>0$ such that:

(i) for any $\lambda \in(0,1)$ and any $\omega$-periodic solution $x$ of the system

$$
\frac{d}{d t}\left[x(t)-\lambda b\left(t, x_{t}\right)\right]=f\left(t, x_{t}\right),
$$

we have $|x(t)|<M$ for $t \in R$;

(ii) $g(u)=: \int_{0}^{\omega} f(s, \hat{u}) d s \neq 0$ for $u \in \partial B_{M}\left(R^{n}\right)$, where $B_{M}\left(R^{n}\right)=\left\{u \in R^{n}\right.$ $:|u|<M\}$, and $\hat{u}$ denotes the constant mapping from $[-\tau, 0]$ to $R^{n}$ with the value $u \in R^{n}$;

(iii) $\operatorname{deg}\left(g, B_{M}\left(R^{n}\right)\right) \neq 0$.

Then there exists at least one $\omega$-periodic solution of the system (2.1) that satisfies $\sup _{t \in R}|x(t)|<M$.

The following remark is introduced by Fang (see Remark 1 in [25]).

Remark 2.1. Theorem A still remains valid if the assumption (ii) is replaced by (ii') there exists a constant $k<1$ such that $|b(t, \phi)-b(t, \varphi)| \leq$ $k\|\phi-\varphi\|$ for $t \in R$ and $\phi, \varphi \in\{\phi \in C:\|\phi\|<M\}$ with $M$ as given in condition (i) of Theorem A.

We will also need the following lemma:

Lemma 2.1 ([3, 20]). Suppose $\sigma \in C_{\omega}^{1}$ and $\sigma^{\prime}(t)<1, t \in[0, \omega]$. Then the function $t-\sigma(t)$ has a unique inverse $\mu(t)$ satisfying $\mu \in C(R, R)$ with $\mu(a+\omega)=\mu(a)+\omega, \forall a \in R$, and if $h \in P C_{\omega}, \sigma^{\prime}(t)<1, t \in[0, \omega]$, then $h(\mu(t)) \in P C_{\omega}$.

Proof. Since $\sigma^{\prime}(t)<1, t \in[0, \omega]$ and $t-\sigma(t)$ is continuous on $R$, it follows that $t-\sigma(t)$ has a unique inverse function $\mu(t) \in C(R, R)$ on $R$. Hence, it suffice to show that $\mu(a+\omega)=\mu(a)+\omega \forall a \in R$. For any $a \in R$, 
by the condition $\sigma^{\prime}(t)<1$, one can find the equation $t-\sigma(t)=a$ exists a unique solution $t_{0}$ and the equation $t-\sigma(t)=a+\omega$ exists a unique solution $t_{1}$, that is,

$$
t_{0}-\sigma\left(t_{0}\right)=a \quad \text { and } \quad t_{1}-\sigma\left(t_{1}\right)=a+\omega
$$

i.e.,

$$
\mu(a)=t_{0}=\sigma\left(t_{0}\right)+a \quad \text { and } \quad \mu(a+\omega)=t_{1} .
$$

As

$$
\begin{aligned}
a+\omega+\sigma\left(t_{0}\right)-\sigma\left(a+\omega+\sigma\left(t_{0}\right)\right) & =a+\omega+\sigma\left(t_{0}\right)-\sigma\left(a+\sigma\left(t_{0}\right)\right) \\
& =a+\omega+\sigma\left(t_{0}\right)-\sigma\left(t_{0}\right) \\
& =a+\omega .
\end{aligned}
$$

It follows that $t_{1}=a+\omega+\sigma\left(t_{0}\right)$. Since $\mu(a+\omega)=t_{1}$, thus, we have

$$
\mu(a+\omega)=t_{1}=a+\omega+\sigma\left(t_{0}\right) \quad \text { and } \quad \mu(a+\omega)=t_{1}=\mu(a)+\omega .
$$

We can easily obtain that if $h \in P C_{\omega}, \tau^{\prime}(t)<1, t \in[0, \omega]$, then $h(\mu(t+\omega))$ $=h(\mu(t)+\omega)=h(\mu(t)), t \in R$, where $\mu(t)$ is the unique inverse function of $t-\tau(t)$, which together with $\mu \in C(R, R)$ implies that $h(u(t)) \in P C_{\omega}$. The proof of Lemma 2.1 is completed.

Lemma 2.2 ([26]). Suppose $x(t)$ is a differently continuous $\omega$-periodic function on $R$ with $(\omega>0)$. Then to any $t_{*} \in R, \max _{t_{*} \leq t \leq t_{*}+\omega}|x(t)| \leq\left|x\left(t_{*}\right)\right|$ $+\frac{1}{2} \int_{0}^{\omega}\left|x^{\prime}(t)\right| d t$

Lemma 2.3. The region $R_{+}^{n}=\left\{N_{i}(t): N_{i}(0)>0, i=1,2, \cdots, n\right\}$ is the positive invariable region of the system (1.1) and (1.2). 
Proof. In view of biological population, we obtain $N_{i}(0)>0$. By the system (1.1), we have

$$
\begin{gathered}
N_{i}(t)=N_{i}(0) \exp \left\{\int _ { 0 } ^ { t } \left[r_{i}(\xi)-\sum_{j=1}^{n} a_{i j}(\xi) N_{j}(\xi)-\sum_{j=1}^{n} b_{i j}(\xi) \int_{-\infty}^{\xi} k_{i j}(\xi-s) N_{j}(s) d s\right.\right. \\
-\sum_{j=1}^{n} c_{i j}(\xi) N_{j}\left(\xi-\tau_{i j}(\xi)\right)-\sum_{j=1}^{n} d_{i j}(\xi) N_{j}^{\prime}\left(\xi-\gamma_{i j}(\xi)\right) \\
\left.\left.-\sum_{j=1}^{n} e_{i j}(\xi) N_{i}(\xi) N_{j}(\xi)\right] d \xi\right\}>0 \\
t \in\left[0, t_{1}\right], i=1,2, \cdots, n,
\end{gathered}
$$

and

$$
\begin{aligned}
& N_{i}(t)= N_{i}\left(t_{k}\right) \exp \left\{\int _ { t _ { k } } ^ { t } \left[r_{i}(\xi)-\sum_{j=1}^{n} a_{i j}(\xi) N_{j}(\xi)-\sum_{j=1}^{n} b_{i j}(\xi) \int_{-\infty}^{\xi} k_{i j}(\xi-s) N_{j}(s) d s\right.\right. \\
&-\sum_{j=1}^{n} c_{i j}(\xi) N_{j}\left(\xi-\tau_{i j}(\xi)\right)-\sum_{j=1}^{n} d_{i j}(\xi) N_{j}^{\prime}\left(\xi-\gamma_{i j}(\xi)\right) \\
&\left.\left.-\sum_{j=1}^{n} e_{i j}(\xi) N_{i}(\xi) N_{j}(\xi)\right] d \xi\right\}>0 \\
& t \in\left(t_{k}, t_{k+1}\right], i=1,2, \cdots, n, k \geq 2, \quad N_{i}\left(t_{k}^{+}\right)=\Delta_{i k} N_{i}\left(t_{k}\right)>0, k \in N, \\
& i=1,2, \cdots, n .
\end{aligned}
$$

Then the solution of (1.1) is positive.

\section{The Main Result}

Since $\tau_{i j}^{\prime}(t)<1, \gamma_{i j}^{\prime}(t)<1, t \in[0, \omega]$, we see that $\tau_{i j}(t), \gamma_{i j}(t)$ all have its inverse function. Throughout the following part, we set $\zeta_{i j}(t), \xi_{i j}(t)$ represent the inverse function of $t-\tau_{i j}(t), t-\gamma_{i j}(t)$, respectively. We also denote 


$$
\Gamma_{i j}(t)=A_{i j}(t)+B_{i j}(t)+\frac{C_{i j}\left(\zeta_{i j}(t)\right)}{1-\tau_{i j}^{\prime}\left(\zeta_{i j}(t)\right)}-\frac{D_{i j}^{\prime}\left(\xi_{i j}(t)\right)}{1-\gamma_{i j}^{\prime}\left(\xi_{i j}(t)\right)} .
$$

Remark 3.1. From Lemma 2.2, we get $\zeta_{i j}(\omega)=\zeta_{i j}(0)+\omega$, $\xi_{i j}(\omega)=\xi_{i j}(0)+\omega, i, j=1,2, \cdots, n$, then

$$
\begin{aligned}
\int_{0}^{\omega} \frac{C_{i j}\left(\zeta_{i j}(s)\right)}{1-\tau_{i j}^{\prime}\left(\zeta_{i j}(s)\right)} d s & =\int_{\zeta_{i j}(0)}^{\zeta_{i j}(\omega)} \frac{C_{i j}(t)\left(1-\tau_{i j}^{\prime}(t)\right)}{1-\tau_{i j}^{\prime}(t)} d t \\
& =\int_{\zeta_{i j}(0)}^{\zeta_{i j}(0)+\omega} C_{i j}(t) d t=\overline{C_{i j}} \omega, \quad i, j=1,2, \cdots, n .
\end{aligned}
$$

Similarly,

$$
\begin{aligned}
\int_{0}^{\omega} \frac{D_{i j}^{\prime}\left(\xi_{i j}(s)\right)}{1-\gamma_{i j}^{\prime}\left(\xi_{i j}(s)\right)} d s & =\int_{\xi_{i j}(0)}^{\xi_{i j}(\omega)} \frac{D_{i j}^{\prime}(t)\left(1-\gamma_{i j}^{\prime}(t)\right)}{1-\gamma_{i j}^{\prime}(t)} d t \\
& =\int_{\xi_{i j}(0)}^{\xi_{i j}(0)+\omega} D_{i j}^{\prime}(t) d t=0, \quad i, j=1,2, \cdots, n .
\end{aligned}
$$

Thus,

$$
\overline{\Gamma_{i j}} \omega=\int_{0}^{\omega} \Gamma_{i j}(t) d t=\left(\overline{A_{i j}}+\overline{B_{i j}}+\overline{C_{i j}}\right) \omega
$$

Theorem 3.1. Suppose that the following conditions hold:

(1) The system of algebraic equations

$$
f(u)=\left(\bar{r}_{i}-\sum_{j=1}^{n}\left(\left(\overline{A_{i j}}+\overline{B_{i j}}+\overline{C_{i j}}\right) \mu_{j}+\overline{E_{i j}} \mu_{i} \mu_{j}\right)\right)_{n \times 1}=0
$$

has a unique positive solution $\mu^{*}=\left(\mu_{1}^{*}, \cdots, \mu_{n}^{*}\right)$;

(2) $\overline{A_{i j}}+\overline{B_{i j}}+\overline{C_{i j}}>0, \overline{r_{i}}>\sum_{j=1, j \neq i}^{n}\left(\overline{A_{i j}}+\overline{B_{i j}}+\overline{C_{i j}}\right) e^{H_{j}}+\sum_{j=1}^{n} \overline{E_{i j}} e^{H_{i}+H_{j}}$, $\tau_{i j}^{\prime}(t)<1, \gamma_{i j}^{\prime}(t)<1$, and $\Gamma_{i j}(t)>0$ 
(3) $K_{0}=: L_{0} e^{M_{0}}<1$.

Then (1.11) and (1.12) has at least one positive $\omega$-periodic solution. Here we have the following denotions:

$$
\begin{aligned}
& \alpha_{i j}=\frac{\Gamma_{i j}^{L}\left(1-\gamma_{i j}^{\prime}\right)^{L}}{\left(1-\gamma_{i j}^{\prime}\right)^{L}+D_{i j}^{M}}, \quad \overline{R_{i}}=\frac{1}{\omega} \int_{0}^{\omega}\left|r_{i}(t)\right| d t, \\
& L_{0}=\max \left\{\sum_{i=1}^{n} \sum_{j=1}^{n}\left|D_{i j}\right|_{0}, \quad \sum_{i=1}^{n} \sum_{j=1}^{n}\left|D_{0, i j}\right|_{0}\right\}, \\
& M_{0}=\max \left\{\sum_{i=1}^{n}\left|\ln \mu_{i}^{*}\right|_{0}, H, \frac{1}{2} \omega \Lambda^{*}+\sum_{i=1}^{n} \Lambda_{i}\right\}, \quad H=\max _{i \in[1, n]}\left\{H_{i}\right\}, \\
& H_{i}=\ln \frac{\bar{r}_{i}}{\alpha_{i i}}+\sum_{j=1}^{n} \frac{\bar{r}_{i}}{\alpha_{i j}}+\left(\overline{R_{i}}+\bar{r}_{i}\right) \omega \\
& \Lambda^{*}=\frac{\sum_{i=1}^{n}\left|r_{i}\right|_{0}+\sum_{i=1}^{n} \sum_{j=1}^{n}\left|A_{i j}\right|_{0} e^{H_{j}}+\sum_{i=1}^{n} \sum_{j=1}^{n}\left|B_{i j}\right|_{0} e^{H_{j}}}{1-\sum_{i=1}^{n} \sum_{j=1}^{n}\left|D_{0, i j}\right|_{0} e^{H_{j}}} \\
& +\frac{\sum_{i=1}^{n} \sum_{j=1}^{n}\left|C_{i j}\right|_{0} e^{H_{j}}+\sum_{i=1}^{n} \sum_{j=1}^{n}\left|E_{i j}\right|_{0} e^{H_{i}+H_{j}}}{1-\sum_{i=1}^{n} \sum_{j=1}^{n}\left|D_{0, i j}\right|_{0} e^{H_{j}}}, \\
& \Lambda_{i}=\max \left\{\left|\ln \frac{r_{i}}{\overline{A_{i i}}+\overline{B_{i i}}+\overline{C_{i i}}}\right|,\right. \\
& \left.\left|\ln \frac{\bar{r}_{i}-\sum_{j=1, j \neq i}^{n}\left(\overline{A_{i j}}+\overline{B_{i j}}+\overline{C_{i j}}\right) e^{H_{j}}-\sum_{j=1}^{n} \overline{E_{i j}} e^{H_{i}+H_{j}}}{\overline{A_{i i}}+\overline{B_{i i}}+\overline{C_{i i}}}\right|\right\},
\end{aligned}
$$

where $\Gamma_{i j}(t)$ are defined by (3.1), and $D_{0, i j}(t)=D_{i j}(t)\left(1-\gamma_{i j}^{\prime}(t)\right)$. 
To prove the above theorem, we make the change of variables

$$
y_{i}(t)=e^{x_{i}(t)}, i=1,2, \cdots, n .
$$

Then the system (1.11) can be rewritten in the following form:

$$
\begin{gathered}
x_{i}^{\prime}(t)=r_{i}(t)-\sum_{j=1}^{n} A_{i j}(t) e^{x_{j}(t)}-\sum_{j=1}^{n} B_{i j}(t) \int_{-\infty}^{t} k_{i j}(t-s) e^{x_{j}(s)} d s-\sum_{j=1}^{n} C_{i j}(t) e^{x_{j}\left(t-\tau_{i j}(t)\right)} \\
-\sum_{j=1}^{n} D_{0 i j}(t) x_{j}^{\prime}\left(t-\gamma_{i j}(t)\right) e^{x_{j}\left(t-\gamma_{i j}(t)\right)}-\sum_{j=1}^{n} E_{i j}(t) e^{x_{i}(t)+x_{j}(t)} \\
i=1,2, \cdots, n .
\end{gathered}
$$

Let $X$ denote the linear space of real value continuous $\omega$-periodic functions on $R$. The linear space $X$ is a Banach space with the usual norm $\|x\|_{0}=\max _{t \in R}|x(t)|=\max _{t \in R} \sum_{j=1}^{n}\left|x_{i}(t)\right|$ for a given $x=\left(x_{1}, \cdots, x_{n}\right) \in X$.

We define the following maps:

$$
\begin{aligned}
& b: R \times C \rightarrow R^{n}, b(t, \phi)=\left(b_{1}(t, \phi), b_{2}(t, \phi), \cdots, b_{n}(t, \phi)\right), \\
& b_{i}(t, \phi)=-\sum_{j=1}^{n} D_{i j}(t) e^{\phi_{j}\left(-\gamma_{i j}(t)\right)} ; \\
& f: R \times C \rightarrow R^{n}, f(t, \phi)=\left(f_{1}(t, \phi), f_{2}(t, \phi), \cdots, f_{n}(t, \phi)\right), \\
& f_{i}(t, \phi)=r_{i}(t)-\sum_{j=1}^{n} A_{i j}(t) e^{\phi_{j}(0)}-\sum_{j=1}^{n} B_{i j}(t) \int_{-\infty}^{0} k_{i j}(t-s) e^{\phi_{j}(s)} d s \\
& \quad-\sum_{j=1}^{n} C_{i j}(t) e^{\phi_{j}\left(-\tau_{i j}(t)\right)}+\sum_{j=1}^{n} D_{i j}^{\prime}(t) e^{\phi_{j}\left(-\gamma_{i j}(t)\right)}-\sum_{j=1}^{n} E_{i j}(t) e^{\phi_{i}(t)+\phi_{j}(t)}, \\
& \quad i=1,2, \cdots, n, \phi=\left(\phi_{1}, \phi_{2}, \cdots, \phi_{n}\right) \in C, t \in R .
\end{aligned}
$$


Clearly, $b: R \times C \rightarrow R^{n}$ and $f: R \times C \rightarrow R^{n}$ are complete continuation functions and system (3.4) takes the form

$$
\frac{d}{d t}\left[x(t)-b\left(t, x_{t}\right)\right]=f\left(t, x_{t}\right)
$$

In the proof of our main result below, we will use the following two important lemmas.

Lemma 3.1. If the assumptions of Theorem 3.1 are satisfied and if $\Omega=\{\phi \in C:\|\phi\|<M\}$, where $M>M_{0}$ is such that $k=L_{0} e^{M}<1$, then

$$
|b(t, \phi)-b(t, \varphi)| \leq k\|\phi-\varphi\|, \text { for } t \in R \text { and } \phi, \varphi \in \Omega \text {. }
$$

Proof. For $t \in R$ and $\phi, \varphi \in \Omega$, we have

$$
\begin{aligned}
\left|b_{i}(t, \phi)-b_{i}(t, \varphi)\right| \leq & \sum_{j=1}^{n} D_{i j}(t)\left|e^{\phi_{j}\left(-\gamma_{i j}(t)\right)}-e^{\varphi_{j}\left(-\gamma_{i j}(t)\right)}\right| \\
\leq & \sum_{j=1}^{n} D_{i j}(t) e^{\sigma_{i j} \phi_{j}\left(-\gamma_{i j}(t)\right)+\left(1-\sigma_{i j}\right) \varphi_{j}\left(-\gamma_{i j}(t)\right)} \\
& \times\left|\phi_{j}\left(-\gamma_{i j}(t)\right)-\varphi_{j}\left(-\gamma_{i j}(t)\right)\right|,
\end{aligned}
$$

for some $\sigma_{i j} \in(0,1)$. Then we get

$$
\left|b_{i}(t, \phi)-b_{i}(t, \varphi)\right| \leq \sum_{j=1}^{n}\left|D_{i j}\right|_{0} e^{M}\|\phi-\varphi\|
$$

Hence,

$$
|b(t, \phi)-b(t, \varphi)| \leq \sum_{i=1}^{n} \sum_{j=1}^{n}\left|D_{i j}\right|_{0} e^{M}\|\phi-\varphi\| \leq L_{0} e^{M}\|\phi-\varphi\|=k\|\phi-\varphi\| .
$$

The proof is thus complete. 
Lemma 3.2. If the assumptions of Theorem 3.1 are satisfied. Then every solution $x \in X$ of the system

$$
\frac{d}{d t}\left[x(t)-\lambda b\left(t, x_{t}\right)\right]=f\left(t, x_{t}\right), \lambda \in(0,1)
$$

satisfies $\|x\|_{0} \leq M_{0}$.

Proof. Let $\frac{d}{d t}\left[x(t)-\lambda b\left(t, x_{t}\right)\right]=f\left(t, x_{t}\right)$ for $x \in X$, that is,

$$
\begin{aligned}
& {\left[x_{i}(t)+\lambda \sum_{j=1}^{n} D_{i j}(t) e^{x_{j}\left(t-\gamma_{i j}(t)\right)}\right]^{\prime}} \\
& =\lambda\left[r_{i}(t)-\sum_{j=1}^{n} A_{i j}(t) e^{x_{j}(t)}-\sum_{j=1}^{n} B_{i j}(t) \int_{-\infty}^{t} k_{i j}(t-s) e^{x_{j}(s)} d s\right. \\
& \left.-\sum_{j=1}^{n} C_{i j}(t) e^{x_{j}\left(t-\tau_{i j}(t)\right)}+\sum_{j=1}^{n} D_{i j}^{\prime}(t) e^{x_{j}\left(t-\gamma_{i j}(t)\right)}-\sum_{j=1}^{n} E_{i j}(t) e^{x_{i}(t)+x_{j}(t)}\right] \\
& \quad i=1,2, \cdots, n ; \lambda \in(0,1)
\end{aligned}
$$

which yields, after integrating from 0 to $\omega$, that

$$
\begin{aligned}
& \int_{0}^{\omega} \sum_{j=1}^{n}\left[A_{i j}(t) e^{x_{j}(t)}+B_{i j}(t) \int_{-\infty}^{t} k_{i j}(t-s) e^{x_{j}(s)} d s+C_{i j}(t) e^{x_{j}\left(t-\tau_{i j}(t)\right)}\right. \\
& \left.\quad+E_{i j}(t) e^{x_{i}(t)+x_{j}(t)}-D_{i j}^{\prime}(t) e^{x_{j}\left(t-\gamma_{i j}(t)\right)}\right] d t \\
& =\int_{0}^{\omega} \sum_{j=1}^{n} \Gamma_{i j}(t) e^{x_{j}(t)} d t+\int_{0}^{\omega} \sum_{j=1}^{n} E_{i j}(t) e^{x_{i}(t)+x_{j}(t)} d t \\
& =\int_{0}^{\omega} r_{i}(t) d t=\bar{r}_{i} \omega, i=1,2, \cdots, n
\end{aligned}
$$


where $\Gamma_{i j}(t)$ is defined by (3.1). From (3.7), we derive

$$
\begin{aligned}
& \int_{0}^{\omega}\left|\left[x_{i}(t)+\lambda \sum_{j=1}^{n} D_{i j}(t) e^{x_{j}\left(-\gamma_{i j}(t)\right)}\right]\right|^{\prime} d t \\
& =\lambda \int_{0}^{\omega} \mid\left[r_{i}(t)-\sum_{j=1}^{n} A_{i j}(t) e^{x_{j}(t)}-\sum_{j=1}^{n} B_{i j}(t) \int_{-\infty}^{t} k_{i j}(t-s) e^{x_{j}(s)} d s\right. \\
& \left.-\sum_{j=1}^{n} C_{i j}(t) e^{x_{j}\left(t-\tau_{i j}(t)\right)}+\sum_{j=1}^{n} D_{i j}^{\prime}(t) e^{x_{j}\left(t-\gamma_{i j}(t)\right)}-\sum_{j=1}^{n} E_{i j}(t) e^{x_{i}(t)+x_{j}(t)}\right] \mid d t \\
& \leq \int_{0}^{\omega}\left|r_{i}(t)\right| d t+\int_{0}^{\omega} \mid \sum_{j=1}^{n}\left[A_{i j}(t) e^{x_{j}(t)}+B_{i j}(t) \int_{-\infty}^{t} k_{i j}(t-s) e^{x_{j}(s)} d s\right. \\
& \left.\quad+C_{i j}(t) e^{x_{j}\left(t-\tau_{i j}(t)\right)}-D_{i j}^{\prime}(t) e^{x_{j}\left(t-\gamma_{i j}(t)\right)}+E_{i j}(t) e^{x_{i}(t)+x_{j}(t)}\right] \mid d t .
\end{aligned}
$$

It follows from (3.7)-(3.9) that

$$
\int_{0}^{\omega} \mid\left[x_{i}(t)+\lambda \sum_{j=1}^{n} D_{i j}(t) e^{x_{j}\left(-\gamma_{i j}(t)\right)}\right]^{\prime} d t \leq\left(\overline{R_{i}}+\overline{r_{i}}\right) \omega, i=1,2, \cdots, n .
$$

By amplifying, it follows (3.8) that

$$
\begin{aligned}
\bar{r}_{i} \omega \geq & \sum_{j=1}^{n} \int_{0}^{\omega} \Gamma_{i j}(t) e^{x_{j}(t)} d t=\sum_{j=1}^{n} \int_{0}^{\omega}\left[\Gamma_{i j}(t) e^{x_{j}(t)}-\left(\alpha_{i j} e^{x_{j}(t)}+\alpha_{i j} D_{i j}(t) e^{x_{j}\left(t-\gamma_{i j}(t)\right)}\right)\right. \\
& \left.+\left(\alpha_{i j} e^{x_{j}(t)}+\alpha_{i j} D_{i j}(t) e^{x_{j}\left(t-\gamma_{i j}(t)\right)}\right)\right] d t \\
= & \sum_{j=1}^{n} \int_{0}^{\omega}\left[\Gamma_{i j}(t) e^{x_{j}(t)}-\left(\alpha_{i j} e^{x_{j}(t)}+\alpha_{i j} D_{i j}(t) e^{x_{j}\left(t-\gamma_{i j}(t)\right)}\right)\right] d t \\
& +\sum_{j=1}^{n} \int_{0}^{\omega}\left[\left(\alpha_{i j} e^{x_{j}(t)}\right.\right. \\
& \left.\left.+\alpha_{i j} D_{i j}(t) e^{x_{j}\left(t-\gamma_{i j}(t)\right)}\right)\right] d t .
\end{aligned}
$$


In view of Remark 3.1, and by a similar analysis, we obtain

$$
\begin{aligned}
\sum_{j=1}^{n} \int_{0}^{\omega}\left[\Gamma_{i j}(t) e^{x_{j}(t)}-\right. & \left.\left(\alpha_{i j} e^{x_{j}(t)}+\alpha_{i j} D_{i j}(t) e^{x_{j}\left(t-\gamma_{i j}(t)\right)}\right)\right] d t \\
& =\sum_{j=1}^{n} \int_{0}^{\omega}\left[\Gamma_{i j}(s)\right. \\
& \left.-\alpha_{i j}-\alpha_{i j} \frac{D_{i j}\left(\xi_{i j}(s)\right)}{1-\gamma_{i j}^{\prime}\left(\xi_{i j}(s)\right)}\right] e^{x_{j}(s)} d t .
\end{aligned}
$$

As $\alpha_{i j}=\frac{\Gamma_{i j}^{L}\left(1-\gamma_{i j}^{\prime}\right)^{L}}{\left(1-\gamma_{i j}^{\prime}\right)^{L}+D_{i j}^{M}}$, it follows that $\Gamma_{i j}(s)-\alpha_{i j}-\alpha_{i j} \frac{D_{i j}\left(\xi_{i j}(s)\right)}{1-\gamma_{i j}^{\prime}\left(\xi_{i j}(s)\right)} \geq 0$.

So we find from (3.11) that

$$
\left.\bar{r}_{i} \omega \geq \sum_{j=1}^{n} \int_{0}^{\omega}\left[\alpha_{i j} e^{x_{j}(t)}+\alpha_{i j} D_{i j}(t) e^{x_{j}\left(t-\gamma_{i j}(t)\right)}\right)\right] d t .
$$

That is,

$$
\left.\bar{r}_{i} \omega \geq \int_{0}^{\omega} \sum_{j=1}^{n}\left[\alpha_{i j} e^{x_{j}(t)}+\alpha_{i j} D_{i j}(t) e^{x_{j}\left(t-\gamma_{i j}(t)\right)}\right)\right] d t .
$$

By the mean value theorem, we see that there exist points $\beta_{i} \in[0, \omega]$, $(i=1, \cdots, n)$ such that

$$
\bar{r}_{i} \geq \sum_{j=1}^{n} \alpha_{i j} e^{x_{j}\left(\beta_{i}\right)}+\sum_{j=1}^{n} \alpha_{i j} D_{i j}\left(\beta_{i}\right) e^{x_{j}\left(\beta_{i}-\gamma_{i j}\left(\beta_{i}\right)\right)}, i=1, \cdots, n,
$$

which implies that

$$
\left.x_{i}\left(\beta_{i}\right) \leq \ln \frac{\bar{r}_{i}}{\alpha_{i i}}, \quad D_{i j}\left(\beta_{i}\right) e^{x_{j}\left(\beta_{i}-\gamma_{i j}\left(\beta_{i}\right)\right)}\right) \leq \frac{\bar{r}_{i}}{\alpha_{i j}}, i=1, \cdots, n .
$$

By (3.10) and (3.14), we can see 


$$
\begin{aligned}
& x_{i}(t)+\lambda \sum_{j=1}^{n} D_{i j}(t) e^{x_{j}\left(t-\gamma_{i j}(t)\right)} \leq x_{i}\left(\beta_{i}\right)+\lambda \sum_{j=1}^{n} D_{i j}\left(\beta_{i}\right) e^{\left.x_{j}\left(\beta_{i}-\gamma_{i j}\left(\beta_{i}\right)\right)\right)} \\
&+\int_{0}^{\omega}\left|\left[x_{i}(t)+\lambda \sum_{j=1}^{n} D_{i j}(t) e^{x_{j}\left(t-\gamma_{i j}(t)\right)}\right]^{\prime}\right| d t \\
& \leq \ln \frac{\overline{r_{i}}}{\alpha_{i i}}+\sum_{j=1}^{n} \frac{\overline{r_{i}}}{\alpha_{i j}}+\left(\overline{R_{i}}+\overline{r_{i}}\right) \omega=: H_{i}, \\
& i=1,2, \cdots, n .
\end{aligned}
$$

For $\lambda \sum_{j=1}^{n} D_{i j}(t) e^{x_{j}\left(t-\gamma_{i j}(t)\right)} \geq 0$, one can find that

$$
x_{i}(t) \leq H_{i}, i=1, \cdots, n .
$$

Besides, from (3.7), we have

$$
\begin{aligned}
x_{i}^{\prime}(t)= & \lambda\left[r_{i}(t)-\sum_{j=1}^{n} A_{i j}(t) e^{x_{j}(t)}-\sum_{j=1}^{n} B_{i j}(t) \int_{-\infty}^{t} k_{i j}(t-s) e^{x_{j}(s)} d s\right. \\
& -\sum_{j=1}^{n} C_{i j}(t) e^{x_{j}\left(t-\tau_{i j}(t)\right)}-\sum_{j=1}^{n} D_{0, i j}(t) x_{j}^{\prime}\left(t-\gamma_{i j}(t)\right) e^{x_{j}\left(t-\gamma_{i j}(t)\right)} \\
& \left.-\sum_{j=1}^{n} E_{i j}(t) e^{x_{i}(t)+x_{j}(t)}\right], i=1,2, \cdots, n .
\end{aligned}
$$

Then by (3.15), we get

$$
\begin{aligned}
\left|x_{i}^{\prime}\right|_{0} \leq & \lambda\left[r_{i}(t)+\sum_{j=1}^{n} A_{i j}(t) e^{x_{j}(t)}+\sum_{j=1}^{n} B_{i j}(t) \int_{-\infty}^{t} k_{i j}(t-s) e^{x_{j}(s)} d s\right. \\
& +\sum_{j=1}^{n} C_{i j}(t) e^{x_{j}\left(t-\tau_{i j}(t)\right)}
\end{aligned}
$$




$$
\begin{aligned}
& \left.+\sum_{j=1}^{n} D_{0, i j}(t) x_{j}^{\prime}\left(t-\gamma_{i j}(t)\right) e^{x_{j}\left(t-\gamma_{i j}(t)\right)}+\sum_{j=1}^{n} E_{i j}(t) e^{x_{i}(t)+x_{j}(t)}\right] \\
\leq & \left|r_{i}\right|_{0}+\sum_{j=1}^{n}\left|A_{i j}\right|_{0} e^{H_{j}}+\sum_{j=1}^{n}\left|B_{i j}\right|_{0} e^{H_{j}}+\sum_{j=1}^{n}\left|C_{i j}\right|_{0} e^{H_{j}} \\
& +\sum_{j=1}^{n}\left|D_{0, i j}\right|_{0}\left|x_{j}^{\prime}\right|_{0} e^{H_{j}}+\sum_{j=1}^{n}\left|E_{i j}\right|_{0} e^{H_{i}+H_{j}}, i=1,2, \cdots, n .
\end{aligned}
$$

Furthermore, we have

$$
\begin{aligned}
\left\|x^{\prime}\right\|_{0}= & \sum_{i=1}^{n}\left|x_{i}^{\prime}\right|_{0} \\
\leq & \sum_{i=1}^{n}\left|r_{i}\right|_{0}+\sum_{i=1}^{n} \sum_{j=1}^{n}\left|A_{i j}\right|_{0} e^{H_{j}}+\sum_{i=1}^{n} \sum_{j=1}^{n}\left|B_{i j}\right|_{0} e^{H_{j}}+\sum_{i=1}^{n} \sum_{j=1}^{n}\left|C_{i j}\right|_{0} e^{H_{j}} \\
& +\sum_{i=1}^{n} \sum_{j=1}^{n}\left|E_{i j}\right|_{0} e^{H_{i}+H_{j}}+\sum_{i=1}^{n} \sum_{j=1}^{n}\left|D_{0, i j}\right|_{0}\left\|x^{\prime}\right\|_{0} e^{H_{j}}
\end{aligned}
$$

By the assumption (3) of Theorem 3.1, we see

$$
\sum_{i=1}^{n} \sum_{j=1}^{n}\left|D_{i j}\right|_{0} e^{H_{j}} \leq \sum_{i=1}^{n} \sum_{j=1}^{n}\left|D_{0, i j}\right|_{0} e^{H} \leq \sum_{i=1}^{n} \sum_{j=1}^{n}\left|D_{0, i j}\right|_{0} e^{M_{0}}<1 .
$$


Then, we have

$$
\begin{gathered}
\|\left. x^{\prime}\right|_{0} \leq \frac{\sum_{i=1}^{n}\left|r_{i}\right|_{0}+\sum_{i=1}^{n} \sum_{j=1}^{n}\left|A_{i j}\right|_{0} e^{H_{j}}+\sum_{i=1}^{n} \sum_{j=1}^{n}\left|B_{i j}\right|_{0} e^{H_{j}}}{1-\sum_{i=1}^{n} \sum_{j=1}^{n}\left|D_{0, i j}\right|_{0} e^{H_{j}}} \\
+\frac{\sum_{i=1}^{n} \sum_{j=1}^{n}\left|C_{i j}\right|_{0} e^{H_{j}}+\sum_{i=1}^{n} \sum_{j=1}^{n}\left|E_{i j}\right|_{0} e^{H_{i}+H_{j}}}{1-\sum_{i=1}^{n} \sum_{j=1}^{n}\left|D_{0, i j}\right|_{0} e^{H_{j}}}=: \Lambda^{*} .
\end{gathered}
$$

By using the extended integral mean value theorem, from (3.8), we can find points $\xi_{i} \in[0, \omega],(i=1, \cdots, n)$ such that

$$
\overline{r_{i}} \omega \geq \sum_{j=1}^{n} \int_{0}^{\omega} \Gamma_{i j}(t) e^{x_{j}(t)} d t=\sum_{j=1}^{n} e^{x_{j}\left(\xi_{j}\right)} \int_{0}^{\omega} \Gamma_{i j}(t) d t, i=1, \cdots, n .
$$

It follows from (3.2) and (3.17) that

$$
\overline{r_{i}} \geq \sum_{j=1}^{n} e^{x_{j}\left(\xi_{j}\right)}\left(\overline{A_{i j}}+\overline{B_{i j}}+\overline{C_{i j}}\right), i=1, \cdots, n
$$

From (3.18), we obtain

$$
e^{x_{i}\left(\xi_{i}\right)}\left(\overline{A_{i i}}+\overline{B_{i i}}+\overline{C_{i i}}\right) \leq \overline{r_{i}}, i=1, \cdots, n .
$$

As $\overline{A_{i i}}+\overline{B_{i i}}+\overline{C_{i i}}>0$, it follows from the above formula that

$$
x_{i}\left(\xi_{i}\right) \leq \ln \frac{\overline{r_{i}}}{\overline{A_{i i}}+\overline{B_{i i}}+\overline{C_{i i}}}, i=1, \cdots, n
$$

On the other hand, there also exists a $\eta_{i} \in[0, \omega]$ such that

$$
x_{i}\left(\eta_{i}\right)=\sup _{t \in[0, \omega]} x_{i}(t), i=1, \cdots, n
$$


it follows from (3.7) that

$$
\begin{aligned}
\overline{r_{i}} & \leq \sum_{j=1}^{n} \overline{\Gamma_{i j}} e^{x_{j}\left(\eta_{i}\right)}+\sum_{j=1}^{n} \overline{E_{i j}} e^{x_{i}\left(\eta_{i}\right)+x_{j}\left(\eta_{i}\right)} \\
& =\sum_{j=1}^{n}\left(\overline{A_{i j}}+\overline{B_{i j}}+\overline{C_{i j}}\right) e^{x_{j}\left(\eta_{i}\right)}+\sum_{j=1}^{n} \overline{E_{i j}} e^{x_{i}\left(\eta_{i}\right)+x_{j}\left(\eta_{i}\right)}, i=1, \cdots, n .
\end{aligned}
$$

From (3.15), we can have

$$
\begin{aligned}
&\left(\overline{A_{i i}}+\overline{B_{i i}}+\overline{C_{i i}}\right) e^{x_{i}\left(\eta_{i}\right)} \\
& \geq \overline{r_{i}}-\sum_{j=1, j \neq i}^{n}\left(\overline{A_{i j}}+\overline{B_{i j}}+\overline{C_{i j}}\right) e^{x_{j}\left(\eta_{i}\right)} \\
&-\sum_{j=1}^{n} \overline{E_{i j}} e^{x_{i}\left(\eta_{i}\right)+x_{j}\left(\eta_{i}\right)} \\
& \geq \overline{r_{i}}-\sum_{j=1, j \neq i}^{n}\left(\overline{A_{i j}}+\overline{B_{i j}}+\overline{C_{i j}}\right) e^{H_{j}}-\sum_{j=1}^{n} \overline{E_{i j}} e^{H_{i}+H_{j}}, i=1, \cdots, n .
\end{aligned}
$$

That is,

$$
x_{i}\left(\eta_{i}\right) \geq \ln \frac{\overline{r_{i}}-\sum_{j=1, j \neq i}^{n}\left(\overline{A_{i j}}+\overline{B_{i j}}+\overline{C_{i j}}\right) e^{H_{j}}-\sum_{j=1}^{n} \overline{E_{i j}} e^{H_{i}+H_{j}}}{\overline{A_{i i}}+\overline{B_{i i}}+\overline{C_{i i}}}, i=1, \cdots, n .
$$

Now, from (3.19) and (3.21), we know that there exist $\delta_{i} \in[0, \omega]$, $(i=1, \cdots, n)$ such that 


$$
\begin{aligned}
& \left|x_{i}\left(\delta_{i}\right)\right| \leq \max \left\{\left|\ln \frac{r_{i}}{\overline{A_{i i}}+\overline{B_{i i}}+\overline{C_{i i}}}\right|,\right. \\
& \left.\left|\ln \frac{\bar{r}_{i}-\sum_{j=1, j \neq i}^{n}\left(\overline{A_{i j}}+\overline{B_{i j}}+\overline{C_{i j}}\right) e^{H_{j}}-\sum_{j=1}^{n} \overline{E_{i j}} e^{H_{i}+H_{j}}}{\overline{A_{i i}}+\overline{B_{i i}}+\overline{C_{i i}}}\right|\right\}=: \Lambda_{i}, i=1, \cdots, n .
\end{aligned}
$$

From (3.16), (3.22) and Lemma 2.2, we have

$$
\left|x_{i}\right| \leq\left|x_{i}\left(\delta_{i}\right)\right|+\frac{1}{2} \int_{0}^{\omega}\left|x_{i}^{\prime}(t)\right| d t \leq \Lambda_{i}+\frac{1}{2} \int_{0}^{\omega}\left|x_{i}^{\prime}\right| d t, i=1, \cdots, n .
$$

Then

$$
\|x\|_{0} \leq \sum_{i=1}^{n}\left|x_{i}\right| \leq \sum_{i=1}^{n} \Lambda_{i}+\frac{1}{2} \int_{0}^{\omega}\left\|x^{\prime}\right\|_{0} d t<\sum_{i=1}^{n} \Lambda_{i}+\frac{1}{2} \Lambda^{*} \omega \leq M_{0} .
$$

Obviously, $M_{0}$ is independent of $\lambda$, the proof is complete.

Based on the above results, we can now apply Theorem A and Remark 2.1 to (3.4) and obtain a proof of Theorem 3.1.

Proof of Theorem 3.1. Obviously, for $M$ as given in Lemma 3.1, condition (1) in Theorem A is satisfied. Let $g(\mu)=\left(g_{1}(\mu), \cdots, g_{n}(\mu)\right)$. Since

$$
\begin{aligned}
g_{i}(\mu)= & \int_{0}^{\omega} f_{i}(s, \hat{u}) d s=\int_{0}^{\omega} r_{i}(t) d t-\sum_{i=1}^{n} \int_{0}^{\omega} A_{i j}(t) d t e^{\mu_{j}}-\sum_{i=1}^{n} \int_{0}^{\omega} B_{i j}(t) d t e^{\mu_{j}} \\
& -\sum_{i=1}^{n} \int_{0}^{\omega} C_{i j}(t) d t e^{\mu_{j}}-\sum_{i=1}^{n} \int_{0}^{\omega} E_{i j}(t) d t e^{\mu_{i}+\mu_{j}} \\
= & \left.\left\{\overline{r_{i}}-\sum_{j=1}^{n}\left[\overline{A_{i j}}+\overline{B_{i j}}+\overline{C_{i j}}\right) e^{\mu_{j}}+\overline{E_{i j}} e^{\mu_{i}+\mu_{j}}\right]\right\} \omega,
\end{aligned}
$$


and $M>\sum_{i=1}^{n}\left|\ln \mu_{i}^{*}\right|$, we have $g(\mu) \neq 0$ for any $\mu \in \partial B_{M}\left(R^{n}\right)$. That is, condition (2) in Theorem A holds. At last, we verify that condition (3) of Theorem A also holds. By assumption (1) of Theorem 3.1 and the formula for the Brouwer degree (see Theorem 2.2.3 in [25]), a straightforward calculation shows that

$$
\begin{aligned}
\operatorname{deg}\left(g, B_{M}\left(R^{n}\right)\right) & =\sum_{\mu \in g^{-1}(0) \cap B_{M}\left(R^{n}\right)} \operatorname{sign} \operatorname{det} \operatorname{Dg}(\mu) \\
& =\operatorname{sign}\left\{(-1)^{n}\left[\operatorname{det}\left[\overline{A_{i j}}+\overline{B_{i j}}+\overline{C_{i j}}+\overline{E_{i j}} e^{\mu_{i}^{*}}\right] e^{\sum_{j=1}^{n} \mu_{j}^{*}}\right\} \neq 0 .\right.
\end{aligned}
$$

By now all the assumptions required in Theorem A hold. It follows from Theorem A and Remark 2.1 that system (3.4) has an $\omega$-periodic solution. Returning to $y_{i}(t)=e^{x_{i}(t)}$, we infer that system (1.8) and (1.9) has at least one positive $\omega$-periodic solution. By Lemma 1.1, we conclude that system (1.1) and (1.2) has at least one positive $\omega$-periodic solution. The proof of Theorem 3.1 is complete.

Consider the following equation:

$$
\begin{aligned}
N_{i}^{\prime}(t)= & N_{i}(t)\left[r_{i}(t)-\sum_{j=1}^{n} a_{i j}(t) N_{j}(t)-\sum_{j=1}^{n} b_{i j}(t) \int_{-\infty}^{t} k_{i j}(t-s) N_{j}(s) d s\right. \\
& -\sum_{j=1}^{n} c_{i j}(t) N_{j}\left(t-\tau_{i j}(t)\right) \\
& \left.-\sum_{j=1}^{n} d_{i j}(t) N_{j}^{\prime}\left(t-\gamma_{i j}(t)\right)-\sum_{j=1}^{n} e_{i j}(t) N_{i}(t) N_{j}(t)\right], i=1,2, \ldots, n,(3
\end{aligned}
$$

which is a special case of system (1.1) without impulse. We get easily the following result. 
Corollary 3.1. Suppose that the following conditions hold:

(4) The system of algebraic equations

$$
f(u)=\left(\overline{r_{i}}-\sum_{j=1}^{n}\left(\left(\overline{a_{i j}}+\overline{b_{i j}}+\overline{c_{i j}}\right) \mu_{j}+\overline{e_{i j}} \mu_{i} \mu_{j}\right)\right)_{n \times 1}=0
$$

has a unique positive solution $\mu^{*}=\left(\mu_{1}^{*}, \cdots, \mu_{n}^{*}\right)$;

$$
\overline{a_{i j}}+\overline{b_{i j}}+\overline{c_{i j}}>0, \overline{r_{i}}>\sum_{j=1, j \neq i}^{n}\left(\overline{a_{i j}}+\overline{a_{i j}}+\overline{a_{i j}}\right) e^{H_{j}^{*}}+\sum_{j=1}^{n} \overline{a_{i j}} e^{H_{i}^{*}+H_{j}^{*}},
$$

$\tau_{i j}^{\prime}(t)<1, \gamma_{i j}^{\prime}(t)<1$ and $\Gamma_{i j}^{*}(t)>0$

(6) $K_{0}^{*}=: L_{0}^{*} e^{M_{0}^{*}}<1$.

Then (3.25) has at least one positive $\omega$-periodic solution. Here we have the following denotions:

$$
\begin{gathered}
\alpha_{i j}^{*}=\frac{\Gamma_{i j}^{* L}\left(1-\gamma_{i j}^{\prime}\right)^{L}}{\left(1-\gamma_{i j}^{\prime}\right)^{L}+d_{i j}^{M}}, \quad R_{i}=\frac{1}{\omega} \int_{0}^{\omega}\left|r_{i}(t)\right| d t, \\
L_{0}^{*}=\max \left\{\sum_{i=1}^{n} \sum_{j=1}^{n}\left|d_{i j}\right|_{0}, \sum_{i=1}^{n} \sum_{j=1}^{n}\left|d_{0, i j}\right|_{0}\right\}, \\
M_{0}^{*}=\max \left\{\sum_{i=1}^{n}\left|\ln \mu_{i}^{*}\right|_{0}, H^{*}, \frac{1}{2} \omega \Delta^{*}+\sum_{i=1}^{n} \Delta_{i}\right\}, \quad H^{*}=\max _{i \in[1, n]}\left\{H_{i}^{*}\right\}, \\
H_{i}^{*}=\ln \frac{\overline{r_{i}}}{\alpha_{i i}^{*}}+\sum_{j=1}^{n} \frac{\overline{r_{i}}}{\overline{\alpha_{i j}^{*}}}+\left(\overline{R_{i}}+\overline{r_{i}}\right) \omega,
\end{gathered}
$$




$$
\begin{gathered}
\Delta^{*}=\frac{\sum_{i=1}^{n}\left|r_{i}\right|_{0}+\sum_{i=1}^{n} \sum_{j=1}^{n}\left|a_{i j}\right|_{0} e^{H_{j}^{*}}+\sum_{i=1}^{n} \sum_{j=1}^{n}\left|b_{i j}\right|_{0} e^{\left.H_{j}^{*}\right)}}{1-\sum_{i=1}^{n} \sum_{j=1}^{n}\left|d_{i j}\right|_{0} e^{H_{j}^{*}}} \\
+\frac{\sum_{i=1}^{n} \sum_{j=1}^{n}\left|c_{i j}\right|_{0} e^{H_{j}^{*}}+\sum_{i=1}^{n} \sum_{j=1}^{n}\left|e_{i j}\right|_{0} e^{H_{i}^{*}+H_{j}^{*}}}{1-\sum_{i=1}^{n} \sum_{j=1}^{n}\left|d_{i j}\right|_{0} e^{H_{j}^{*}}}, \\
\Delta_{i}=\max \left\{\left|\ln \frac{r_{i}}{\overline{a_{i i}}+\overline{b_{i i}}+\overline{c_{i i}}}\right| \mid \operatorname{In} \frac{\left.\bar{r}_{i=1, j \neq i}-\overline{\sum_{i j}}+\overline{B_{i j}}+\overline{c_{i j}}\right) e^{H_{j}^{*}}-\sum_{j=1}^{n} \overline{e_{i j}} e^{H_{i}^{*}+H_{j}^{*}}}{\overline{a_{i i}}+\overline{b_{i i}}+\overline{c_{i i}}}\right. \\
\Gamma_{i j}^{*}(t)=a_{i j}(t)+b_{i j}(t)+\frac{c_{i j}\left(\zeta_{i j}(t)\right)}{1-\tau_{i j}^{\prime}\left(\zeta_{i j}(t)\right)}-\frac{d_{i j}^{\prime}\left(\xi_{i j}(t)\right)}{1-\gamma_{i j}^{\prime}\left(\xi_{i j}(t)\right)}, \\
d_{0, i j}(t)=d_{i j}(t)\left(1-\gamma_{i j}^{\prime}(t)\right),
\end{gathered}
$$

and $\zeta_{i j}(t), \xi_{i j}(t)$ represent the inverse function of $t-\tau_{i j}(t), t-\gamma_{i j}(t)$ $(i, j=1,2, \cdots, n)$, respectively.

Proof. Its proof is similar to the proof of Theorem 3.1. Here we omit it. Similarly, we can get the following results. 
Theorem 3.2. Let us assume that conditions of Theorem 3.1 hold, and then the conclusion of Theorem 3.1 holds for the following system:

$$
\left\{\begin{aligned}
N_{i}^{\prime}(t)= & -N_{i}(t)\left[r_{i}(t)-\sum_{j=1}^{n} a_{i j}(t) N_{j}(t)-\sum_{j=1}^{n} b_{i j}(t) \int_{-\infty}^{t} k_{i j}(t-s) N_{j}(s) d s\right. \\
& -\sum_{j=1}^{n} c_{i j}(t) N_{j}\left(t-\tau_{i j}(t)\right)-\sum_{j=1}^{n} d_{i j}(t) N_{j}^{\prime}\left(t-\gamma_{i j}(t)\right) \\
& \left.-\sum_{j=1}^{n} e_{i j}(t) N_{i}(t) N_{j}(t)\right], i=1,2, \ldots, n, t \neq t_{k}, \\
\Delta N_{i}\left(t_{k}\right)= & N_{i}\left(t_{k}^{+}\right)-N_{i}\left(t_{k}\right)=\left(p_{i k}+q_{i k}\right) N_{i}\left(t_{k}\right), i=1,2, \cdots, n, \quad k=1,2, \ldots
\end{aligned}\right.
$$

Proof. Its proof is similar to the proof of Theorem 3.1. Here we omit it.

Corollary 3.2. Let us assume that conditions of Corollary 3.1. hold, and then the conclusion of Corollary 3.1. holds for the following system:

$$
\begin{gathered}
N_{i}^{\prime}(t)=-N_{i}(t)\left[r_{i}(t)-\sum_{j=1}^{n} a_{i j}(t) N_{j}(t)-\sum_{j=1}^{n} b_{i j}(t) \int_{-\infty}^{t} k_{i j}(t-s) N_{j}(s) d s\right. \\
\left.-\sum_{j=1}^{n} c_{i j}(t) N_{j}\left(t-\tau_{i j}(t)\right)-\sum_{j=1}^{n} d_{i j}(t) N_{j}^{\prime}\left(t-\gamma_{i j}(t)\right)-\sum_{j=1}^{n} e_{i j}(t) N_{i}(t) N_{j}(t)\right], \\
i=1,2, \ldots, n .
\end{gathered}
$$

Proof. Its proof is similar to the proof of Theorem 3.1. Here we omit it.

\section{Applications}

In order to illustrate some features of our main result, in the following, we will apply Theorem 3.1 to some special cases which have been studied extensively in the literature. 
Application 4.1. We consider an $n$-species neutral delay competition system in a periodic environment with impulse:

$$
\left\{\begin{array}{l}
N_{i}^{\prime}(t)= \pm N_{i}(t)\left[a_{i}(t)-\sum_{j=1}^{n} b_{i j}(t) N_{j}(t)-\sum_{j=1}^{n} c_{i j}(t) N_{j}\left(t-\tau_{i j}(t)\right)\right. \\
\left.\quad-\sum_{j=1}^{n} d_{i j}(t) N_{j}^{\prime}\left(t-\gamma_{i j}(t)\right)-\sum_{j=1}^{n} e_{i j}(t) N_{i}(t) N_{j}(t)\right], i=1,2, \ldots, n, t \neq t_{k}, \\
\Delta N_{i}\left(t_{k}\right)=\left(p_{i k}+q_{i k}\right) N_{i}\left(t_{k}\right), i=1,2, \cdots, n, \quad k=1,2, \ldots,
\end{array}\right.
$$

where $\quad b_{i j}, c_{i j}, e_{i j} \in C(R,[0,+\infty)) d_{i j} \in C^{1}(R,[0,+\infty)), \tau_{i j} \in C^{1}(R, R)$, $\gamma_{i j} \in C^{2}(R, R)$ are continuous $\omega$-periodic functions. $a_{i} \in C(R, R)$ are continuous $\omega$-periodic functions with $\int_{0}^{\omega} a_{i}(t) d t>0$. Applying our Theorem 3.1 to Equation (4.1), we can obtain the following theorem.

Theorem 4.1. Assume that the following conditions are satisfied:

(1) The system of algebraic equations

$$
\left.g(u)=\left(\overline{a_{i}}-\sum_{j=1}^{n}\left(\overline{B_{i j}}+\overline{C_{i j}}\right) \mu_{j}+\overline{E_{i j}} \mu_{i} \mu_{j}\right)\right)_{n \times 1}=0,
$$

has a unique positive solution $\mu^{*}=\left(\mu_{1}^{*}, \cdots, \mu_{n}^{*}\right)$;

(2) $\overline{B_{i j}}+\overline{C_{i j}}>0, \overline{a_{i}}>\sum_{j=1, j \neq i}^{n}\left(\overline{B_{i j}}+\overline{C_{i j}}\right) e^{H_{j}}+\sum_{j=1}^{n} \overline{E_{i j}} e^{H_{i}+H_{j}}, \tau_{i j}^{\prime}(t)<1$, $\gamma_{i j}^{\prime}(t)<1$, and $\Theta_{i j}(t)>0$

(3) $K_{1}=: L_{1} e^{M_{1}}<1$. 
Then (4.1) has at least one positive $\omega$-periodic solution. Here we have the following denotions:

$$
\begin{aligned}
& \alpha_{i j}=\frac{\Theta_{i j}^{L}\left(1-\gamma_{i j}^{\prime}\right)^{L}}{\left(1-\gamma_{i j}^{\prime}\right)^{L}+D_{i j}^{M}}, \quad L_{1}=\max \left\{\sum_{i=1}^{n} \sum_{j=1}^{n}\left|D_{i j}\right|_{0}, \quad \sum_{i=1}^{n} \sum_{j=1}^{n}\left|D_{0, i j}\right|_{0}\right\}, \\
& M_{1}=\max \left\{\sum_{i=1}^{n}\left|\ln \mu_{i}^{*}\right|_{0}, H^{*}, \frac{1}{2} \omega \Lambda^{*}+\sum_{i=1}^{n} \Lambda_{i}\right\}, \quad H^{*}=\max _{i \in[1, n]}\left\{H_{i}^{*}\right\}, \\
& H_{i}^{*}=\ln \frac{\overline{a_{i}}}{\alpha_{i i}}+\sum_{j=1}^{n} \frac{\overline{a_{i}}}{\alpha_{i j}}+\left(\overline{A_{i}}+\overline{a_{i}}\right) \omega \\
& \Lambda^{*}=\frac{\sum_{i=1}^{n}\left|a_{i}\right|_{0}+\sum_{i=1}^{n} \sum_{j=1}^{n}\left|B_{i j}\right|_{0} e^{H_{j}^{*}}+\sum_{i=1}^{n} \sum_{j=1}^{n}\left|C_{i j}\right|_{0} e^{H_{j}^{*}}+\sum_{i=1}^{n} \sum_{j=1}^{n}\left|E_{i j}\right|_{0} e^{H_{i}^{*}+H_{j}^{*}}}{1-\sum_{i=1}^{n} \sum_{j=1}^{n}\left|D_{0, i j}\right|_{0} e^{H_{j}^{*}}} . \\
& \Lambda_{i}=\max \left\{\left|\ln \frac{a_{i}}{\overline{B_{i i}}+\overline{C_{i i}}}\right|,\left|\ln \frac{\overline{a_{i}}-\sum_{j=1, j \neq i}^{n}\left(\overline{B_{i j}}+\overline{C_{i j}}\right) e^{H_{j}^{*}}-\sum_{j=1}^{n} \overline{E_{i j}} e^{H_{i}^{*}+H_{j}^{*}}}{\overline{B_{i i}}+\overline{C_{i i}}}\right|\right\}, \\
& \Theta_{i j}(t)=B_{i j}(t)+\frac{C_{i j}\left(\rho_{i j}(t)\right)}{1-\tau_{i j}^{\prime}\left(\rho_{i j}(t)\right)}-\frac{D_{i j}^{\prime}\left(\varrho_{i j}(t)\right)}{1-\gamma_{i j}^{\prime}\left(\varrho_{i j}(t)\right)},
\end{aligned}
$$

where $\rho_{i j}(t), \varrho_{i j}(t)$ represent the inverse function of $t-\sigma_{i j}(t), t-\tau_{i j}(t)$, respectively, and

$$
\begin{aligned}
\overline{A_{i}}=\frac{1}{\omega} \int_{0}^{\omega}\left|a_{i}(t)\right| d t, \quad & B_{i j}(t)=b_{i j}(t) \prod_{0<t_{k}<t}\left(1+p_{i k}+q_{i k}\right), \\
C_{i j}(t) & =c_{i j}(t) \prod_{0<t_{k}<t-\tau_{i j}(t)}\left(1+p_{i k}+q_{i k}\right),
\end{aligned}
$$




$$
\begin{gathered}
D_{i j}(t)=d_{i j}(t) \prod_{0<t_{k}<t-\gamma_{i j}(t)}\left(1+p_{i k}+q_{i k}\right), \quad D_{0, i j}(t)=D_{i j}(t)\left(1-\gamma_{i j}^{\prime}(t)\right), \\
E_{i j}(t)=e_{i j}(t) \prod_{0<t_{k}<t}\left(1+p_{i k}+q_{i k}\right)\left(1+p_{j k}+q_{j k}\right), \tau=\max \left\{\tau_{i j}(t), \gamma_{i j}(t)\right\}, \\
i, j=1,2, \cdots, n .
\end{gathered}
$$

Remark 4.1. When $i=1$, or $\tau_{i j}(t)=\sigma_{i j}(t)$ and $p_{i k}+q_{i k}=0$ we can derive some immediate corollaries of Theorem 4.1. On the other hand, we can see that our Theorem 4.1 can hold without the assumption $\bar{a}_{i}>0$. When $\bar{a}_{i}<0$ Xia's main result (see Theorem 3.1 in [19]) cannot be applied. Therefore, comparing with [19], our result improves and generalizes the results in [19].

Application 4.2. We consider the single specie neutral delay logistic equation with impulse:

$$
\left\{\begin{aligned}
\frac{d N}{d t} & = \pm N(t)\left[a(t)-b(t) N(t)-\sum_{i=1}^{n} c_{i}(t) \int_{-\infty}^{t} k_{i}(t-s) N(s) d s\right. \\
& \left.-\sum_{j=1}^{m} d_{j}(t) N\left(t-\sigma_{j}(t)\right)-\sum_{l=1}^{p} e_{l}(t) N^{\prime}\left(t-\tau_{l}(t)\right)\right], t \neq t_{k} \\
N\left(t_{k}^{+}\right)= & \left(p_{k}+q_{k}\right) N\left(t_{k}\right), k=1,2, \ldots
\end{aligned}\right.
$$

where $\quad b, c_{i}, d_{j} \in C(R,[0,+\infty)), e_{l} \in C^{1}(R,[0,+\infty)), \sigma_{j} \in C^{1}(R, R)$, $\tau_{l} \in C^{2}(R, R)$ are continuous $\omega$-periodic functions. $a \in C(R, R)$ are continuous $\omega$-periodic functions with $\int_{0}^{\omega} a(t) d t>0$. Applying our Theorem 3.1 to Equation (4.2), we can obtain the following theorem.

Theorem 4.2. Assume that the following conditions are satisfied:

(1) $\bar{B}+\sum_{i=1}^{n} \overline{C_{i}}+\sum_{j=1}^{m} \overline{D_{j}}>0, \tau_{i j}^{\prime}(t)<1, \gamma_{i j}^{\prime}(t)<1$ and $\Gamma_{i}(t)>0$; 
(2) $K_{2}=: L_{2} e^{M_{2}}<1$.

Then (4.1) has at least one positive $\omega$-periodic solution. Here we have the following denotions:

$$
\begin{aligned}
& \bar{A}=\frac{1}{\omega} \int_{0}^{\omega}|a(t)| d t, B(t)=b(t) \prod_{0<t_{k}<t}\left(1+p_{k}+q_{k}\right), C_{i}(t)=c_{i}(t) \prod_{0<t_{k}<t}\left(1+p_{k}+q_{k}\right), \\
& D_{j}(t)=d_{j}(t) \prod_{0<t_{k}<t-\sigma_{j}(t)}\left(1+p_{k}+q_{k}\right), E_{l}(t)=e_{l}(t) \prod_{0<t_{k}<t-\tau_{l}(t)}\left(1+p_{k}+q_{k}\right), \\
& E_{0, l}(t)=E_{l}(t)\left(1-\tau_{l}^{\prime}(t)\right), \quad \alpha=\frac{\Gamma_{1}^{L}\left(1-\tau_{l}^{\prime}\right)^{L}}{\left(1-\tau_{l}^{\prime}\right)^{L}+E_{l}^{M}}, \\
& L_{2}=\max \left\{\sum_{l=1}^{p}\left|E_{l}\right|_{0}, \sum_{l=1}^{p}\left|E_{0, l}\right|_{0}\right\}, M_{2}=\max \left\{H^{*}, \Delta^{*}\right\}, H^{*}=\ln \frac{\bar{a}}{\alpha}+\frac{\bar{a}}{\alpha}+(\bar{A}+\mid \bar{a}) \omega, \\
& \Delta^{*}=\frac{\omega}{2} \frac{|a|_{0}+\left(|B|_{0}+\sum_{i=1}^{n}\left|C_{i}\right|_{0}+\sum_{j=1}^{m}\left|D_{j}\right|_{0}\right) e^{H^{*}}}{1-\left|E_{0, l}\right|_{0} e^{H^{*}}}+\left|\ln \frac{\bar{a}}{\bar{B}+\sum_{i=1}^{n} \overline{C_{i}}+\sum_{j=1}^{m} \overline{D_{j}}}\right| \\
& \Gamma_{1}(t)=B(t)+\sum_{i=1}^{n} C_{i}(t)+\sum_{j=1}^{m} \frac{D_{j}\left(\mu_{j}(t)\right)}{1-\sigma^{\prime}\left(\mu_{j}(t)\right)}-\sum_{l=1}^{p} \frac{E_{l}^{\prime}\left(\nu_{l}(t)\right)}{1-\tau_{l}^{\prime}\left(\nu_{l}(t)\right)},
\end{aligned}
$$

and $\mu_{j}(t), \nu_{l}(t)$ represent the inverse function of $t-\sigma_{j}(t), t-\tau_{l}(t)$, respectively.

Remark 4.2. When $i=0, j=l=1, \sigma_{j}(t)=\tau_{l}(t)$, and $p_{k}+q_{k}=0$, we can derive an immediate corollary of Theorem 4.2 , which is also an answer to the open problem 9.2 due to Kuang [1]. On the other hand, we can see that our Theorem 4.2 can hold without the assumption $\bar{a}>0$. When $\bar{a}<0$, Wang's main result (see Theorem 3.1 in [20]) cannot be applied. Therefore, comparing with [20], our result improves and generalizes the results in [20]. 


\section{Acknowledgement}

The research was supported by the project of research study and innovative experiment of college students in Hunan (No. CX1504), the Scientific Research Fund of Hunan Provincial Education Department (No. 17A030), the training target of the young backbone teachers in Hunan colleges and Universities (No. [2015]361), the Science and Technology Plan Project of Hunan Province (No. 2016TP1020), and the construct program of the key discipline in Hunan Province.

\section{References}

[1] Y. Kuang, Delay Differential Equations with Applications in Population Dynamics, Academic Press, New York, 1993.

[2] H. Fang and J. B. Li, On the existence of periodic solutions of a neutral delay model of single-species population growth, J. Math. Anal. Appl. 259 (2001), 8-17.

[3] S. P. Lu and W. G. Ge, Existence of positive periodic solutions for neutral population model with multiple delays, Appl. Math. Comput. 153 (2004), 885-892.

[4] Z. H. Yang and J. D. Cao, Positive periodic solutions of neutral Lotka-Volterra system with periodic delays, Appl. Math. Comput. 149 (2004), 661-687.

[5] Z. J. Liu and L. S. Chen, On positive periodic solutions of a non-autonomous neutral delay $n$-species competitive system, Nonlinear Analysis 68 (2008), 1409-1420.

[6] J. R. Yan and A. M. Zhao, Oscillation and stability of linear impulsive delay differential equations, J. Math. Anal. Appl. 227 (1998), 187-194.

[7] X. Z. Liu and G. Ballinger, Boundedness for impulsive delay differential equations and applications to population growth models, Nonlinear Anal. 53 (2003), 1041-1062.

[8] B. C. Zhang and Y. J. Liu, Global attractivity for certain impulsive delay differential equations, Nonlinear Analysis 35 (2003), 725-736.

[9] J. Zhen, Z. E. Ma and M. A. Han, The existence of periodic solutions of the $n$-species Lotka-Voterra competition systems with impulsive, Chaos Solutions Fractals 22 (2004), 181-188.

[10] L. Z. Dong and L. S. Chen, A periodic predator-prey-chain system with impulsive perturbation, J. Comput. Appl. Math. 223 (2009), 578-584.

[11] L. F. Nie, Z. D. Teng, L. Hu and J. G. Peng, Qualitative analysis of a modified LeslieGower and Holling-type II predator-prey model with state dependent impulsive effects, Nonlinear Analysis: RWA. 11 (2010), 1364-1373.

[12] A. M. Samoilenko and N. A. Perestyuk, Differential Equations with Impulse Effect, World Scientific Publisher, Singapore, 1993. 
[13] D. D. Bainov and P. Simeonov, Impulsive Differential Equations: Periodic Solutions and Applications, Longman, England, 1993.

[14] A. M. Samoilenko and N. A. Perestyuk, Impulsive Differential Equations, World Scientific, Singapore, 1995.

[15] M. Benchohra, J. Henderson and S. K. Ntouyas, Impulsive Differential Equations and Inclusions, Hindawi Publishing Corporation, Volume 2, New York, 2006.

[16] J. Zhen, Z. Ma and M. Han, The existence of periodic solutions of the $n$-species Lotka-Volterra competition systems with impulse, Chaos Solitons Fractals 22 (2004), 181-188.

[17] H. F. Huo, Existence of positive periodic solutions of a neutral delay Lotka-Volterra systems with impulses, Comput. Math. Appl. 48 (2004), 1833-1846.

[18] Y. K. Li, L. H. Lu and X. Y. Zhu, Existence of periodic solutions in $n$-species foodchain system with impulsive, Nonlinear Analysis: RWA. 7 (2006), 414-431.

[19] Y. H. Xia, Positive periodic solutions for a neutral impulsive delayed Lotka-Volterra competition system with the effect of toxic substance, Nonlinear Analysis: RWA. 8 (2007), 204-221.

[20] Q. Wang and B. X. Dai, Existence of positive periodic solutions for a neutral population model with delays and impulse, Nonlinear Analysis 69 (2008), 3919-3930.

[21] M. X. He and F. D. Chen, Dynamic behaviors of the impulsive periodic multi-species predator-prey system, Comput. Math. Appli. 57 (2009), 248-265.

[22] R. Q. Shi and L. S. Chen, An impulsive predator-prey model with disease in the prey for integrated pest management, Communications in Nonlinear Science and Numerical Simulation 5 (2010), 421-429.

[23] L. Erbe, W. Krawcewicz and J. H. Wu, A composite coincidence degree with applications to boundary value problems of neutral equations, Trans. Amer. Math. Soc. 335 (1993), 459-478.

[24] W. Krawcewicz and J. H. Wu, Theory of Degrees with Applications to Bifurcations and Differential Equations, John Wiley \& Sons, Inc., New York, 1996.

[25] H. Fang, Positive periodic solutions of $n$-species neutral delay systems, Czechoslovak Math. J 53 (2003), 561-570.

[26] Q. Wang, B. X. Dai and Y. M. Chen, Multiple periodic solutions of an impulsive predator-prey model with Holling IV functional response, Math. Comput. Model. 49 (2009), 1829-1836. 